\title{
Oral contraceptives and venous thromboembolism: old questions revisited
}

\author{
Jürgen Dinger
}

\section{Background}

Two studies recently published in the British Medical Journal, a retrospective cohort study using information from the Danish registries ${ }^{1}$ and the MEGA case-control study for which cases were identified via specialised anticoagulation clinics in The Netherlands, ${ }^{2}$ revisit the question of whether so-called 'third generation' combined oral contraceptives (COCs) containing the progestogens, desogestrel and gestodene, increase the risk of venous thromboembolism (VTE) more than so-called 'second generation' COCs as represented by products containing levonorgestrel. It now seems that this old dispute is being extended to other progestogens. These studies found an increased relative risk for desogestrel and gestodene, as well as for cyproterone acetate and drospirenone, compared to levonorgestrel. In addition, the MEGA casecontrol study found an increased relative risk for lynestrenol and norgestimate. For the sake of brevity this commentary focuses on drospirenone, which is currently the most widely used progestogen. Many of the following arguments, however, apply also to other progestogens.

An increased risk of VTE has been linked to COCs since the 1960s, and has been associated with the estrogen dose. In 1995, World Health Organization (WHO) investigators alerted the public that desogestrel and gestodene carry a higher risk of VTE than levonorgestrel. In the ensuing years several studies were published, some of which supported and some of which conflicted with the WHO results. The heated scientific debate led to a widespread 'Pill-scare' propagated in the media, and ultimately occupied the law courts. In 2002, the High Court of Justice in London came to the conclusion that "there is not as a matter of probability any increased relative risk of VTE carried by any of the third generation oral contraceptives $\ldots$ as compared with second generation products containing levonorgestrel". A legal decision, however, cannot be a substitute for scientific judgment. Even 14 years after the publication of the WHO study, whether the increased relative risk for desogestrel and gestodene is real or the result of bias and confounding remains controversial.

In addition to the above-mentioned Danish cohort study and the MEGA case-control study, three other large studies $^{3-5}$ have investigated the VTE risk of drospirenone in comparison to levonorgestrel or other progestogens. Even a cursory comparison shows that the results of all of these studies, although using different methodologies, are not fundamentally different.

\section{Study results}

The crude incidence rate ratios or odds ratios for drospirenone compared to levonorgestrel or other

J Fam Plann Reprod Health Care 2009; 35(4): 211-213

Berlin Center for Epidemiology and Health Research, Berlin, Germany

Jürgen Dinger, MD, PhD, Director

Correspondence to: Dr Jürgen Dinger, Berlin Center for Epidemiology and Health Research, Invalidenstrasse 115, 10115 Berlin, Germany. E-mail: dinger@zeg-berlin.de progestogens in the Danish cohort study, ${ }^{1}$ the MEGA casecontrol study, ${ }^{2}$ the EURAS study ${ }^{3}$ (a large, multi-national, prospective cohort study with active surveillance study participants), the Ingenix study 4 (a prospective cohort study in a USA claims database using propensity score matching) and the German case-control study 5 (using outpatient offices from the primary care sector as well as specialised diagnostic centres) were $1.35,1.04,1.14,0.97$ and 0.95 , respectively. The corresponding adjusted risk estimates were $1.64,1.7,1.04,0.97$ and 0.97 [risk estimates and $95 \%$ confidence intervals (CIs) are shown in Figure 1].

All five studies were observational. In observational research the possibility of bias and residual confounding can never be entirely eliminated, and the ability to infer causation is correspondingly limited.6,7 A point in the gradient of declining relative risk is always reached at which the risk estimate becomes so small that the impact of bias and residual confounding cannot be reliably assessed. ${ }^{8}$ Put another way, the resolving power of the 'epidemiologic microscope' is limited, and risk estimates in the range of 0.5 to 2.0 usually do not allow differentiation between causation as against bias and confounding.

For this reason alone it is clear that the relative risk estimates for drospirenone are similar and the available evidence does not allow the conclusion that drospirenone is associated with an increased risk of VTE compared to levonorgestrel. Overall, an impressively large amount of scientific data is available and it is unlikely that new observational studies would change this overall assessment. It is even doubtful whether a large randomised clinical trial could resolve any remaining uncertainty about potential small increases in the relative risk, since it is possible that differential dropout rates and partial unblinding due to different pharmacological profiles (e.g. effects on acne and premenstrual symptoms) would make it difficult to interpret small relative risks.

These general considerations are not meant to discourage the reader from critically comparing the available studies. All of them had methodological limitations. Rather than attempt to list all the shortcomings, this commentary focuses on two issues that are sufficient to explain the differences between the relative risk estimates.

Lidegaard ${ }^{9}$ and Dunn ${ }^{10}$ stressed the similarities in results between the Danish cohort study and the MEGA case-control study. The relative risk estimates for drospirenone relative to levonorgestrel were similar. However, there were noteworthy differences among the studies regarding the relative risk estimates for current COC use vs non-use (Danish cohort study, 2.8; MEGA case-control study, 5.0) as well as the early use effect for levonorgestrel (see below).

Study limitations and confounding effects The two existing case-control studies cannot contribute much to this debate. The results of the MEGA case-control study were not statistically significant for the comparison of drospirenone (and most of the other progestogens) and levonorgestrel and thus cannot establish an increased relative risk for drospirenone. The second case-control study has been published only as an abstract.

Returning now to the cohort studies, which are more informative. An important point in the three cohort studies 


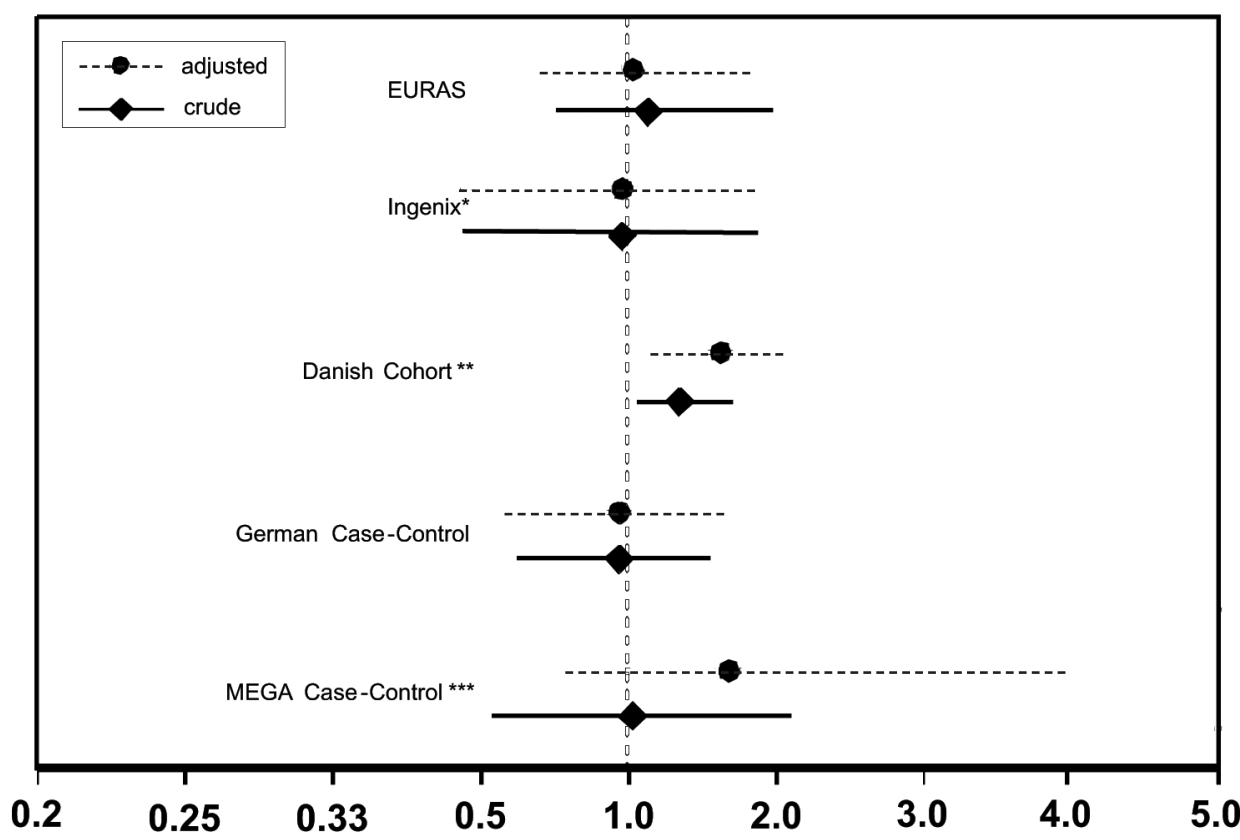

Figure 1 Relative risk estimates and $95 \%$ confidence intervals from five studies on the venous thromboembolism risk associated with the use of drospirenone-containing combined oral contraceptives relative to levonorgestrel or other progestogens including levonorgestrel (Ingenix study) 4

*No adjusted risk estimates calculated because of propensity score matching; comparison vs other progestogens including levonorgestrel.

${ }^{*}$ Crude risk estimate and $95 \%$ confidence intervals (Cls) are based on the author's own calculations derived from Table 2 in the Danish cohort study. ${ }^{1}$ ${ }^{* * *}$ Crude risk estimate and $95 \% \mathrm{Cls}$ are based on the author's own calculations derived from Table 3 in the MEGA casecontrol study. ${ }^{2}$

\section{2} 0.25 0.33 0.5

1.0

2.0

3.0 5.0

is the availability and control of confounder information. The Ingenix study, which found no increased risk (hazard ratio, 0.97), used propensity score matching to achieve a close balance on the patient characteristics available from the automated claims database. Individuals with similar propensity scores based on medical history as well as socioeconomic factors probably also have similarities in factors that are not part of the claims database [e.g. body mass index (BMI)]. However, it is difficult to substantiate these assumed similarities in the database.

The Danish cohort study analysed datasets that included the entire Danish female population of fertile age. The investigators are to be congratulated for conducting such a large study that also adjusted for the confounder information assessable in the Danish registries. However, information on relevant risk factors such as BMI and family history was not available. The investigators assumed "that bias as a result of failing to control for body mass index and family predisposition was small, if present at all". However, given the small magnitude of differences interpreted by the investigators, the failure to control for those confounding factors cannot be ignored. In fact, the EURAS study, a large field study that included almost 60000 COC users, showed beyond reasonable doubt that the studied drospirenone-containing COC was preferentially prescribed to overweight and obese women thus demonstrating the importance of controlling for this effect. Furthermore, the study demonstrated that the combination of risk factors (e.g. obesity and family history of VTE) led to an approximate multiplicative risk of VTE, which further emphasises the need to control for potential differences among COC users.

Two secular trends in Denmark during the study period (1995-2005) have an important bearing on the interpretation of the reported results. (1) Prescriptions of levonorgestrel-containing COCs declined substantially, while prescriptions of drospirenone increased steadily following market introduction in 2001. (2) Obesity in Danish women increased dramatically during the study period. Thus the well-known preferential prescribing of drospirenone to obese COC users was more important than suggested by the authors. It is likely that this lack of relevant confounder information contributed to the increased relative risk estimates for drospirenone.

The crude risk estimates in the EURAS and the Danish cohort studies were similar. Adjustment in the latter study, which lacked information on BMI and family history of VTE, increased the risk estimates, whereas in the EURAS study adjustment for these confounders decreased the risk estimates (see Figure 1).

A more important limitation of the Danish cohort study was left censorship of the prescription data. To illustrate: since exposure data in Denmark were only available from 1995, a woman who used an 'old' progestogen such as levonorgestrel for 4.5 years before 1995, and thereafter during follow-up for an additional 6 months, would have been classified incorrectly as a short-term user (i.e. 6 months of use), when in fact she was a long-term user (i.e. correct duration of use was 5 years). By contrast, the duration of use for a woman who used a new progestogen such as drospirenone from 2001 to 2005 would have been correctly recorded as 5 years. This differential misclassification is important, as COCs increase VTE risk maximally during the first months of use, after which the risk declines. Therefore long-term users have a substantially lower risk of VTE than short-term users. Thus in the Danish study the misclassification of long-term users as short-term users gave rise to an incorrectly low incidence rate of VTE for shortterm use of levonorgestrel. Accordingly, the investigators of the Danish cohort study - unlike the EURAS or MEGA case-control investigators - were not able to demonstrate an early use effect for levonorgestrel. As a result they overestimated the relative risk for drospirenone (and other progestogens) compared to levonorgestrel.

Due to the massive size of this study, virtually all of its comparisons were statistically robust; nevertheless its ability to infer causation based on small differences was clearly limited. Statistical significance in this study did not equate with causation and it only showed that the difference between the treatments is not due to chance. Bias and residual confounding remain plausible alternative explanations of the study results.

Both the EURAS and the Ingenix studies were postauthorisation safety studies conducted as Phase IV commitments to the United States Food and Drug Administration and the European Medicines Agency (EMEA). The studies were nevertheless criticised by some as being industry-sponsored, as well as not sufficiently powered to identify small relative risks. As for sponsorship, the studies were mandated by the 
authorities, and the study protocols and the statistical analysis plans were reviewed by the authorities as well as by independent safety monitoring and advisory boards. In both studies the cases were subjected to blinded adjudication by clinicians. In the EURAS study all reported cases of VTE, confirmed as well as unconfirmed, were sent to the authorities for review. With this level of transparency the focus should be on the scientific aspects of the studies rather than industry sponsorship. As for statistical power, both studies were large (each comprised about 60000 women) and sufficiently robust in statistical terms to detect meaningful differences in relative risk estimates (see Figure 1).

\section{Concluding remarks}

In conclusion: the existing evidence continues to suggest that the risk of VTE attributable to COCs is a class effect, primarily dependent on the dose of estrogen. The perpetuation of the debate about the existence or nonexistence of small differences in risk attributed to individual progestogens will not lead to a consensus among the scientific community as long as the discussion is based on observational studies.

\section{Statements on funding and competing interests}

Funding None identified.

Competing interests The author was actively involved in the conduct of the EURAS and the German case-control studies; both were post-authorisation safety studies for European health authorities and were funded by the manufacturer of drospirenone (Bayer Schering Pharma AG).

\section{References}

1 Lidegaard Ø, Løkkegaard E, Svendsen AL, Agger C. Hormonal contraception and risk of venous thromboembolism: national follow-up study. BMJ 2009; 339: b2890.

2 Van Hylckama Vlieg A, Helmerhorst FM, Vandenbroucke JP, Doggen CJM, Rosendaal FR. Effects of oestrogen dose and progestogen type on venous thrombotic risk associated with oral contraceptives: results of the MEGA case-control study. BMJ 2009; 339: b2921.

3 Dinger JD, Heinemann LAJ, Kühl-Habich D. The safety of a drospirenone-containing oral contraceptive: final results from the European active surveillance study on oral contraceptives based on 142,475 women-years of observation. Contraception 2007; 75: 344-354.

4 Seeger JD, Loughlin J, Eng PM, Clifford CR, Cutone J, Walker AM. Risk of thromboembolism in women taking ethinylestradiol/drospirenone and other oral contraceptives. Obstet Gynecol 2007; 110: 587-593.

5 Dinger JC, Voigt K, Moehner S. Case-control study: use of oral contraceptives containing dienogest and risk of venous thromboembolism. Pharmacoepidemiol Drug Saf 2009; 18: S114.

6 Susser M. What is a cause and how do we know one? A grammar for pragmatic epidemiology. Am J Epidemiol 1991; 133: 635-648.

7 Grimes DA, Schulz KF. Bias and causal associations in observational research. Lancet 2002; 359: 248-252.

8 Shapiro S. Bias in the evaluation of low-magnitude associations: an empirical perspective. Am J Epidemiol 2000; 151: 939-945.

9 Lidegaard $\varnothing$. COCs and VTE risks. http://www.bmj.com/cgi/ eletters/339/aug13_2/b2890\#218855 [Accessed 1 September 2009].

10 Dunn N. Oral contraceptives and venous thromboembolism. BMJ 2009; 339: b3164.

FICTION BOOK REVIEW

Tomorrow. Graham Swift. London, UK: Picador, 2008, ISBN-13: 978-0-33045-026-3. Price: £7.99. Pages: 256 (paperback)

On a summer's night, Paula lies awake contemplating her incipient 50th birthday and reflecting on 16 years of family life and parenthood. So far, so many parallels with my own and no doubt the lives of many other readers. But it soon becomes clear that she is also worrying about a major challenge, a family milestone, that lies ahead tomorrow for Paula, Mike and their 16-year-old twins, Kate and Nick.

Paula and Mike met as students in Brighton, in the 'Swinging Sixties'. Their days together come across as happy times developing independence from their very differing but equally challenging upbringings. They settle down, marry, and both find themselves in good jobs, comfortable, contented, but lacking children in their lives

Swift explores themes of relationships, fidelity, bereavement and the impact of infertility, lineage and parenthood. A black cat called Otis plays an unexpected, if somewhat unlikely, central role in the development of Paula and

We hope that journal readers enjoyed reading Tomorrow, and also discovering whether their opinion of the book matched that of our guest reviewer. In the January 2010 issue, the fiction book under scrutiny will be The Whole Day Through by Patrick Gale (288 pages, Fourth Estate, 2009, ISBN-13: 978-000730-601-5).

We want to remind journal readers that if they would like to offer to review an appropriate fiction title of their own choosing then they should contact the Journal Editorial Office by e-mail (journal@fsrh.org) in the first instance with details of their nominated title.
Mike's family story. Without children, a kindly elderly neighbour suggests they adopt a stray cat which fills an apparent void in their lives. When Otis returns from a long, mysterious and muchmourned absence, Paula finds herself unexpectedly confiding in her sympathetic local vet, with perhaps predictable consequences.

The book is written in an elegiac yet compulsive style. Whilst it is unlikely to be considered the poolside hit of the summer, its rhythm and reflective prose placed a gentle grip on my night-time reading routine. I liked the sense of climax that builds gradually; however, a clinical audience is unlikely to be surprised by the underlying theme, once revealed. Tomorrow usefully explores a neglected aspect of fertility, in addition to the more usual territory: the ethica and moral dilemmas thrown up by modern social attitudes to long-term relationships and childlessness. It reminds us that people respond to these challenges as individuals, often in an unexpected way, and that as clinical practitioners this can be hard to predict, both in the immediate and longer term.

Perhaps because the narration lies entirely with Paula, the book fails to properly explore some of the other interesting issues her family have faced: the biologist who is confronted with unexplained infertility and the impact of this on lineage and heritage; the reactions of the twins themselves on learning the truth; the role of the veterinary surgeon with his more prosaic approach to conception. Some readers may find the self-absorption and analytical approach of the narrator irritating. But I feel that the book reminds us that, for many people, these are big and pertinent problems that don't always fade with the passage of time. As technology and science advance, we are likely to find ourselves grappling with ever more complex puzzles, and wondering at the variety of the human response.

In the end, I was a little disappointed by this book. It promised much at the beginning but ultimately it wallowed too much in the Paula perspective. More from Mike would have provided more food for thought, and even some valuable insight into masculine attitudes to parenthood, biological or otherwise.

Reviewed by Imogen Stephens, MD, FFSRH Consultant in Public Health Medicine and Associate Editor, Journal of Family Planning and Reproductive Health Care

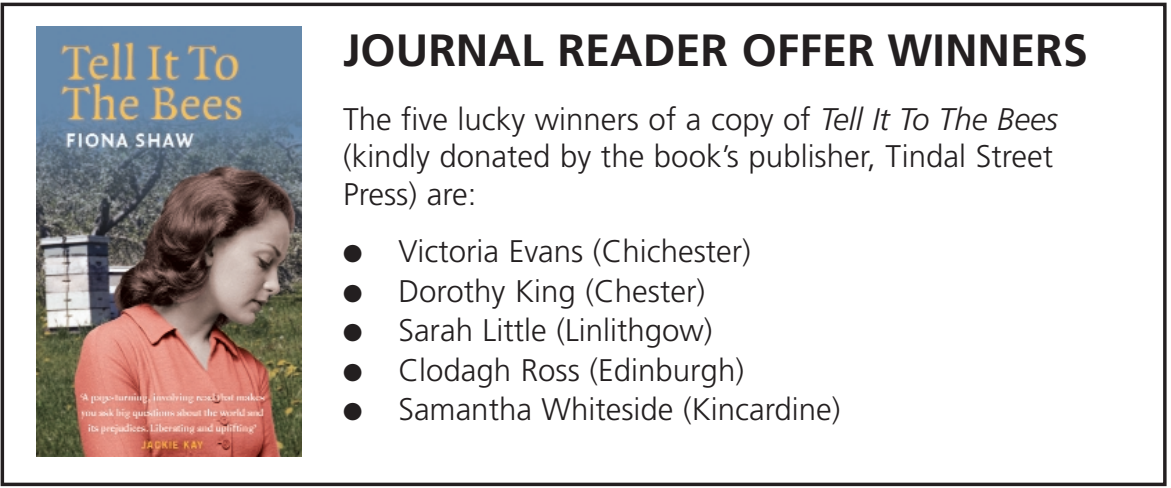

